\title{
Residuos $\beta$-lactámicos y tetraciclinas en la leche fresca adquirida por Comités de Vaso de Leche de los distritos de San Jerónimo y Andahuaylas, Apurímac, Perú
}

\author{
$\beta$-lactam and tetracycline residues in fresh milk acquired by the Glass of Milk \\ Committees of the San Jerónimo and Andahuaylas districts in Apurímac, Peru
}

David Choque-Quispe ${ }^{1,4}$, Mirian E. Obregon-Yupanqui ${ }^{1}$, Carlos A. LigardaSamanez ${ }^{1}$, Betsy S. Ramos-Pacheco', Julio C. Sichez-Muñoz ${ }^{1}$, Aydeé M. SolanoReynoso $^{2}$, Yudith Choque-Quispe ${ }^{3}$

\section{Resumen}

\begin{abstract}
El objetivo del trabajo fue evaluar el efecto de la presencia de residuos de antibióticos $\beta$-lactámicos y tetraciclinas, así como las propiedades fisicoquímicas de la leche fresca adquirida por los Comités de Vaso de Leche de dos distritos de Apurímac, Perú. Se analizaron muestras de leche por triplicado, provenientes de 37 y 32 comités de los distritos de San Jerónimo y Andahuaylas, respectivamente. Se determinaron las propiedades fisicoquímicas: densidad, acidez, $\mathrm{pH}$, sólidos totales (ST) y prueba de alcohol, las cuales se compararon con las Normas Técnicas Peruanas (NTP) para leche fresca. La presencia de compuestos antibióticos $\beta$-lactámicos y tetraciclinas se determinó a través del kit colorimétrico Betastar ${ }^{\circledR}$. Las propiedades fisicoquímicas se correlacionaron con la presencia de antibióticos a través del coeficiente de Pearson. Se realizó un análisis de componentes principales (ACP). El 10.8\% de las muestras de leche provenientes de los comités de San Jerónimo fue positivo para tetraciclina, mientras que el 9.4 y $3.1 \%$ de los comités de San Jerónimo y Andahuaylas, respectivamente, fueron positivos para $\beta$ lactámicos. Un alto procentaje de las muestras de leche estuvieron fuera de los rangos
\end{abstract}

${ }^{1}$ Escuela Profesional de Ingeniería Agroindustrial, Facultad de Ingeniería, Universidad Nacional José María Arguedas, Andahuaylas, Apurímac, Perú

${ }^{2}$ Escuela Profesional de Ingeniería Ambiental, Universidad Tecnológica de los Andes, Andahuaylas, Apurimac, Perú

${ }^{3}$ Escuela Profesional de Agronomía, Universidad Nacional de San Antonio Abad del Cusco, Cusco, Perú

${ }^{4}$ E-mail: davidchoqueq@gmail.com

Recibido: 19 de enero de 2019

Aceptado para publicación: 20 de enero de 2020

Publicado: 11 de agosto de 2020 
establecidos por las NTP para densidad, pH, acidez y ST. Del análisis ACP se desprende que la acidez y la densidad son las principales variables que pemitirían determinar la presencia de antibioticos.

Palabras clave: antibioticos, $\beta$-lactámicos, tetraciclinas, leche fresca, comités de vaso de leche

\section{ABSTRACT}

The aim of this study was to evaluate the effect of the presence of residues of $\beta$ lactam antibiotics and tetracyclines, as well as the physicochemical properties of fresh milk acquired by the Glass of Milk Committees of two districts of Apurimac, Peru. Triplicate milk samples were analyzed from 37 and 32 committees in the districts of San Jerónimo and Andahuaylas respectively. Density, acidity, $\mathrm{pH}$, total solids (TS) and alcohol test were determined and compared with the Peruvian Technical Standards (NTP) for fresh milk. The presence of $\beta$-lactam and tetracycline antibiotic compounds was determined through the Betastar ${ }^{\circledR}$ colorimetric kit. The physicochemical properties were correlated with the presence of antibiotics through the Pearson coefficient. A principal component analysis (PCA) was performed. Results showed that $10.8 \%$ of the milk samples from the San Jerónimo committees were positive for tetracycline, while 9.4 and 3.1\% of the San Jerónimo and Andahuaylas committees, respectively, were positive for $\beta$-lactams. A high percentage of milk samples were outside the ranges established by the NTP for density, $\mathrm{pH}$, acidity and TS. The PCA analysis shows that acidity and density are the main variables that would would determine the presence of antibiotics.

Key words: antibiotic, $\beta$-lactams, tetracyclines, fresh milk, milk glass committees

\section{INTRODUCCIÓN}

El gobierno peruano, a través de la historia, ha establecido como política de desnutrición y atención escolar, la dotación de leche fresca a través de los programas sociales (Diez-Canseco y Saavedra-Garcia, 2017; Camos, 2018), con el objetivo de mejorar el nivel nutricional de las poblaciones en situación de pobreza, así como contribuir a mejorar la calidad de vida de esta población, que por su precaria situación económica no estaría en condiciones de atender sus necesidades elementales. Estas acciones se realizan a través de las muncipalidades y los comités de compra de leche fresca (Briones y Peña, 2018). Estos comités son organizaciones lideradas generalmente por mujeres madres, que tienen por objetivo mejorar el estado nutricional y la calidad de vida de los más pobres a través de la entrega de leche para los desayunos, teniendo como prioridad los niños menores 6 años, gestantes y madres que dan de lactar, así como a niños entre 7 y 13 años, adultos mayores y pacientes con tuberculosis (Camos, 2018).

La leche es un producto de comercialización cotidiana, que por su alto valor nuticional es imprescindible en la la dieta humana; sin embargo, deben considerarse los aspectos de aseguramiento de la calidad fisicoquímica y microbiológica de este producto, la cuales están establecidos en las Normas Técnicas Peruanas (NTP), el Decreto Supremo No 007-2017-MINAGRI, Perú (MINAGRI, 2017), y de manera más global en el CODEX Alimentarius (2011) para leche y productos lácteos. 
Enfermedades en las vacas lecheras, principalemente la mastitis, se refleja en la calidad de su leche (Molina et al., 2010). Las vacas afectadas con mastitis son usualmente tratadas con antibioticos, los cuales pueden pasar a la leche (Llanos, 2002). El empleo de antibióticos, sin tomar en cuenta las dosis ni periodos de aplicación, conlleva a la formación de microrganismos resistentes (Prado et al., 2002; Gutiérrez et al., 2005; Máttar et al., 2009).

La mastitis es tratada generalmente de forma fácil, rápida y barata con la aplicación intramamaria de antibióticos $\beta$-lactámicos (Guerrero, 2002; Salas et al., 2013). Una parte de la dosis administrada es absorbida y pasa al torrente sanguíneo, otra parte es inactivada por la leche y los productos generados por la infección y, el resto, que es la mayor parte, es excretada en la leche durante los ordeños siguientes, siendo los animales de baja producción los que se tardan más en eliminar el fármaco (Molina et al., 2010). Por tanto, el ordeño frecuente ayuda a diluir el antibiótico y acorta el tiempo de eliminación de este (Molina et al., 2010; Fernández, 2012).

Los residuos de antibióticos en leche son perjudiciales para la salud pública así como en los procesos de la industria láctea. En cuanto a la salud, son capaces de producir toxicidad de tipo crónica, causar reacciones alérgicas de distintas magnitudes y tienen efectos carcinógenos; además pueden causar la reducción de la síntesis de vitaminas (Monge et al., 1993; Ramírez et al., 2001; Brunton et al., 2011). Los residuos de antibióticos $\beta$-lactámicos pueden provocar reacciones adversas como erupciones maculopapulares, urticaria, fiebre, broncoespasmo, vasculitis, dermatitis exfoliativa y anafilaxia en distintos grados, pudiendo causar graves reacciones en personas hipersensibles (Noa-Lima et al., 2009; Camacho, et al., 2010), mientras que la leche con residuos de tetraciclinas pueden provocar reacciones adversas (Brunton et al., 2011).
El objetivo del trabajo fue evaluar el efecto de residuos $\beta$-lactámicos y tetraciclinas en las propiedades fisicoquímicas de la leche fresca adquirida por los Comités de Vaso de Leche de los distritos de San Jerónimo y Andahuaylas, Apurímac, Perú.

\section{Materiales y Métodos}

Las muestras de leche fresca a granel (1 litro) provinieron de 69 comités del programa vaso de leche: 37 del distrito de Andahuaylas y 32 de San Jerónimo, ambos ubicados en la región de Apurímac, Perú. El muestreo fue inopinado con previa solicitud de autorización por parte de los comités de vaso de leche. Las muestras de leche fueron recolectadas al azar a las 07:00 horas durante los meses de julio y agosto de 2018 y llevadas de inmediato al laboratorio bajo refrigeración en una cámara con hielo. Los análisis fueron desarrollados en las instalaciones del Laboratorio de Calidad y Química de la Escuela Profesional de Ingeniería Agroindustrial de la Universidad Nacional José María Arguedas, Andahuaylas, Perú.

La determinación de la presencia de antibióticos $\beta$-lactámicos y tetraciclinas se realizó a través de un método cromatográfico, para lo cual se tomó $10 \mathrm{ml}$ de leche fresca a temperatura ambiente, siguiendo el protocolo propuesto para el kit Betastar ${ }^{\circledR}$ Combo «S» (Neogen, USA), que determina la presencia o ausencia del residuo de ambos antibióticos.

En el caso de los parámetros fisicoquímicos de la leche, la densidad fue determinada con un termo-lactodensímetro Quevenne $15-40$ y expresada en $\mathrm{g} / \mathrm{ml} \mathrm{a} 15^{\circ} \mathrm{C}$, la acidez como porcentaje de ácido láctico de determinó a través de titulación, el $\mathrm{pH}$ a $15{ }^{\circ} \mathrm{C}$ con un potenciómetro HANNA HI 5522, los sólidos totales (ST) fueron expresados en gramos de sólidos por $100 \mathrm{~g}$ de muestra, y la prueba de alcohol para la verificación de contaminación por mastitis. En todos los casos se siguió los protocolos establecidos en las 
Normas Técnicas Peruana (NTP, 2003). Las determinaciones se realizaron por triplicado en todos los casos.

Los datos de los parámetros fisicoquímicos fueron analizados considerando los límites de calidad establecidos en el CODEX Alimentarius (2011) y el Decreto Supremo N. ${ }^{\circ}$ 007-2017-MINAGRI, Perú (MINAGRI, 2017). Se aplicó la correlación de Pearson con relación a la presencia de antibióticos, que derivó en un Análisis de Componentes Principales - ACP. El análisis de datos se realizó con un nivel de significancia del 5\%,y se utilizó el software Minitab v17.

\section{Resultados y Discusión}

El $49.3 \%$ de los comités presentaron valores de densidad de la leche por debajo del límite inferior - LI (1.0296 g/ml) (Cuadro 1), mientras que la acidez se encuentra en un rango entre 0.09 y $0.22 \mathrm{~g}$ ácido láctico/ $100 \mathrm{~g}$, denotando una alta variabilidad (18.1\%), y observándose que el $42.0 \%$ de los comités presento valores anómalos para este parámetro. Así mismo, el $\mathrm{pH}$ de la leche del $79.7 \%$ de los comités presentó valores fuera de los rangos establecidos por el CODEX Alimentarius (2011), y el 100\% de las muestras contenía valores muy por debajo del valor límite inferior de sólidos totales $(11.4 \mathrm{~g} / 100 \mathrm{~g})$. La leche del $37 \%$ de los comités fueron positivos a la prueba de alcohol.

El $10.1 \%$ de las muestras de leche resultó positivo para tetraciclinas y $1.4 \%$ fue positivo para $\beta$-lactamicos (Cuadro 2). El uso intensivo de antibióticos se observa en zonas productoras de leche. Ortiz et al. (2008) reportó que el 89 y $62 \%$ de muestras de leche fresca proveniente de la cuenca de Arequipa, Perú contenían $\beta$-lactámicos y tetraciclinas, respectivamente. Así mismo, Chávez (2013) encontró que el $2.7 \%$ de muestras de leche fresca provenientes de la costa central del Perú y destinada a la elaboración de productos lácteos contenia antibióticos, en tanto que Carrasco y Obando (2013) encontró 17.2\%

Cuadro 1. Características fisicoquímicas de la leche fresca provenientes de los Comités del Vaso de Leche de 37 distritos de Andahuaylas y 32 distritos de San Jerónimo (Apurímac, Perú, 2018)

\begin{tabular}{lccccccc}
\hline Parámetros & Media $\pm \mathrm{s}$ & Mínimo & Máximo & $\begin{array}{c}\text { C.V. } \\
(\%)\end{array}$ & $\begin{array}{c}\text { Comités } \\
\text { fuera del } \\
\text { lite }\end{array}$ & LI & LS \\
\hline $\begin{array}{l}\text { Densidad } \\
\text { (g/ml) }\end{array}$ & $\begin{array}{c}1.0287 \pm \\
0.0036\end{array}$ & 1.0200 & 1.0340 & 0.4 & 49.3 & 1.0296 & 1.034 \\
$\begin{array}{l}\text { Acidez } \\
(\mathrm{g} \mathrm{AL} / 100 \mathrm{~g})\end{array}$ & $0.16 \pm 0.029$ & 0.09 & 0.22 & 18.1 & 42.0 & 0.13 & 0.17 \\
$\mathrm{pH}$ & $6.41 \pm 0.11$ & 6.16 & 6.65 & 1.6 & 79.7 & 6.5 & 6.8 \\
$\mathrm{ST}(\mathrm{g} / 100 \mathrm{~g})$ & $7.89 \pm 0.95$ & 5.91 & 9.41 & 12.0 & 100.0 & 11.4 & --- \\
\hline
\end{tabular}

LI: Límite inferior; LS: Límite superior; C.V.: Coeficiente de variación; AL: ácido láctico 
Cuadro 2. Presencia de antibióticos en la leche fresca provenientes de los Comités del Vaso de Leche de 37 distritos de Andahuaylas y 32 distritos de San Jerónimo (Apurímac, Perú, 2018)

\begin{tabular}{|c|c|c|c|c|c|}
\hline \multirow{3}{*}{ Distrito } & \multirow{3}{*}{$\begin{array}{l}\text { Total de } \\
\text { comités }\end{array}$} & \multicolumn{4}{|c|}{ Positivo } \\
\hline & & \multicolumn{2}{|l|}{ Tetraciclina } & \multicolumn{2}{|c|}{$\beta$-lactamicos } \\
\hline & & Comité & $\%$ & Comité & $\%$ \\
\hline \multirow[t]{4}{*}{ San Jerónimo } & 37 & Ccantupata & 10.8 & & 0 \\
\hline & & Chaccarapata & & & \\
\hline & & Chullcuisa & & & \\
\hline & & San Juan de Yañecc & & & \\
\hline \multirow[t]{3}{*}{ Andahuaylas } & 32 & Tapaya & 9.4 & Ccoñeccpuquio II & 3.1 \\
\hline & & Tablina & & & \\
\hline & & Yunca Alta & & & \\
\hline Total & 69 & 7 & 10.1 & 1 & 1.4 \\
\hline
\end{tabular}

de muestras de leche con residuos de $\beta$ lactámicos y tetraciclinas en puntos de expendio de la ciudad de Chiclayo, Perú. Reportes similares existen en la literatura científica, como son los casos de Matiguas, Nicaragua (Rodriguez y Mendoza, 2011) y Machala, Ecuador (Aroca, 2016).

La presencia de antibióticos en la leche fresca no evidencia una relación significativa con los parámetros bajo estudio, puesto que los valores del coeficiente de correlación $r_{s}$ son muy bajos (Cuadro 3).

La correlación general de los parámetros de la leche fresca proveniente de los comités (Cuadro 4), evidencia que la densidad presenta una baja correlación positiva con los antibióticos $\beta$-lactámicos y tetraciclínicos ( 0.13 y 0.12 , respectivamente). De igual manera, los componentes tetraciclínicos presentan baja correlación positiva con la acidez,
Cuadro 3. Correlación de parámetros de la leche con presencia de antibióticos en leche fresca provenientes de los Comités del Vaso de Leche (Apurímac, Perú, 2018)

\begin{tabular}{|c|c|c|c|}
\hline \multicolumn{2}{|c|}{ Parámetro } & \multirow{2}{*}{$\begin{array}{c}\begin{array}{c}\text { Tetra- } \\
\text { ciclinas }\end{array} \\
-0.25\end{array}$} & \multirow{2}{*}{$\begin{array}{c}\begin{array}{c}\beta- \\
\text { lactámicos }\end{array} \\
0.25\end{array}$} \\
\hline Densidad & $r_{s}$ & & \\
\hline \multirow{3}{*}{ Acidez } & p-value & 0.56 & 0.56 \\
\hline & $r_{s}$ & 0.08 & -0.08 \\
\hline & p-value & 0.84 & 0.84 \\
\hline \multirow[t]{2}{*}{$\mathrm{pH}$} & $r_{s}$ & -0.08 & 0.08 \\
\hline & p-value & 0.85 & 0.85 \\
\hline \multirow[t]{2}{*}{ ST } & $r_{s}$ & -0.08 & 0.08 \\
\hline & p-value & 0.85 & 0.85 \\
\hline \multirow{2}{*}{$\begin{array}{l}\text { Prueba } \\
\text { de } \\
\text { alcohol }\end{array}$} & $r_{s}$ & 0.38 & -0.38 \\
\hline & p-value & 0.36 & 0.36 \\
\hline
\end{tabular}


Cuadro 4. Correlación general de las variables de las muestras de leche cruda provenientes de los Comités del Vaso de Leche de 37 distritos de Andahuaylas y 32 distritos de San Jerónimo (Apurímac, Perú, 2018)

\begin{tabular}{lccccccc}
\hline Variables & \multicolumn{1}{c}{ Densidad Acidez } & $\mathrm{pH}$ & $\mathrm{ST}$ & $\begin{array}{c}\text { Prueba } \\
\text { de } \\
\text { alcohol }\end{array}$ & Tetraciclina $\beta$-Lactámicos \\
\hline Densidad & 1.00 & 0.39 & 0.29 & 0.98 & 0.12 & 0.13 & 0.12 \\
Acidez & 0.39 & 1.00 & -0.17 & 0.36 & 0.23 & 0.16 & 0.03 \\
pH & 0.29 & -0.17 & 1.00 & 0.34 & -0.21 & -0.04 & 0.00 \\
Sólidos totales & 0.98 & 0.36 & 0.34 & 1.00 & 0.08 & 0.13 & 0.11 \\
P. de alcohol & 0.12 & 0.23 & -0.21 & 0.08 & 1.00 & 0.13 & -0.09 \\
Tetraciclinas & 0.13 & 0.16 & -0.04 & 0.13 & 0.13 & 1.00 & -0.04 \\
$\beta$-lactámicos & 0.12 & 0.03 & 0.00 & 0.11 & -0.09 & -0.04 & 1.00 \\
\hline
\end{tabular}

Cuadro 5. Categorización de parámetros de la leche cruda en componentes principales

\begin{tabular}{lccccccc}
\hline Variables & PC 1 & PC 2 & PC 3 & PC 4 & PC 5 & PC 6 & PC 7 \\
\hline Densidad & $\mathbf{0 . 9 6}$ & -0.10 & 0.00 & -0.09 & 0.00 & -0.23 & 0.09 \\
Acidez & $\mathbf{0 . 5 4}$ & 0.52 & 0.19 & -0.13 & -0.45 & 0.43 & 0.00 \\
pH & 0.34 & -0.71 & -0.36 & 0.05 & 0.22 & 0.45 & 0.00 \\
Sólidos totales & $\mathbf{0 . 9 6}$ & -0.15 & -0.03 & -0.07 & 0.00 & -0.21 & -0.09 \\
Prueba de alcohol & 0.19 & $\mathbf{0 . 6 8}$ & -0.11 & -0.27 & 0.64 & 0.10 & 0.00 \\
Tetraciclinas & 0.25 & $\mathbf{0 . 4 0}$ & -0.28 & 0.84 & 0.02 & -0.01 & 0.00 \\
B-lactámicos & 0.15 & -0.20 & $\mathbf{0 . 8 8}$ & 0.29 & 0.27 & 0.10 & 0.00 \\
\hline
\end{tabular}

ST y prueba de alcohol $(0.16,0.13$ y 0.13 , respectivamente). Por otro lado, se aprecia alta correlación entre ST y densidad $\left(r_{s}\right.$ $=0.98$ ), aunque esta es moderada con la acidez y $\mathrm{pH}(0.36$ y 0.34 , respectivamente). Así mismo, la baja correlación negativa entre el $\mathrm{pH}$ y la acidez de la leche $\left(r_{s}=-0.17\right)$ puede deberse a la posibilidad de que se esté adulterando la leche con la adición de agua.
La correlación negativa entre el $\mathrm{pH}$ y la prueba de alcohol $\left(r_{s}=-.021\right)$ es indicativo de que las muestras de leche que resultaron negativas a la prueba de alcohol presentan mayores valores de $\mathrm{pH}$. Alvarado et al. (2006) menciona que una leche «no-ácida» y de buena calidad puede resultar positiva a esta prueba, debido entre otras razones, a un desbalance mineral, principalmente de calcio. 


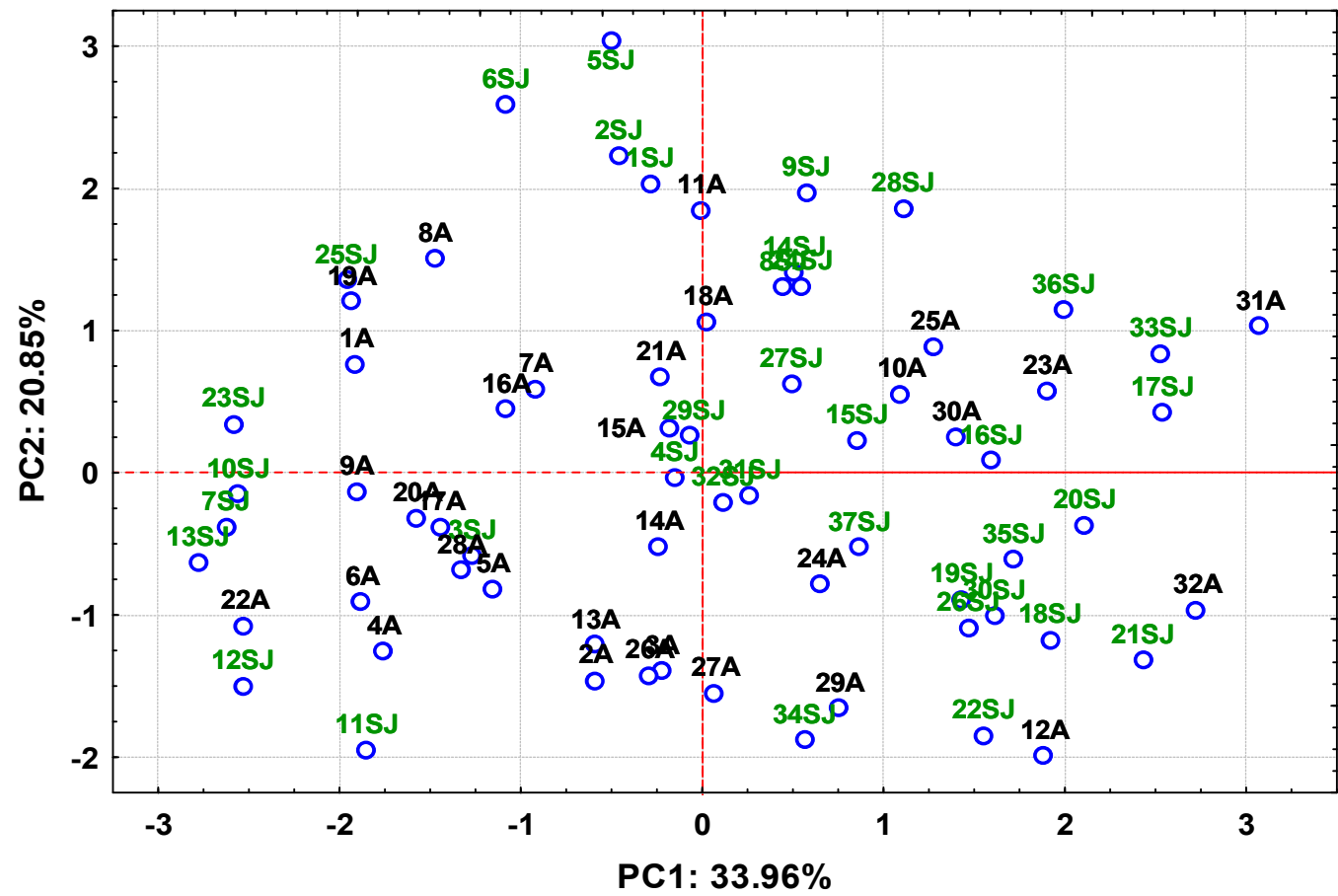

Figura 1. Gráfico de puntuaciones (Scores) para PC1 y PC2

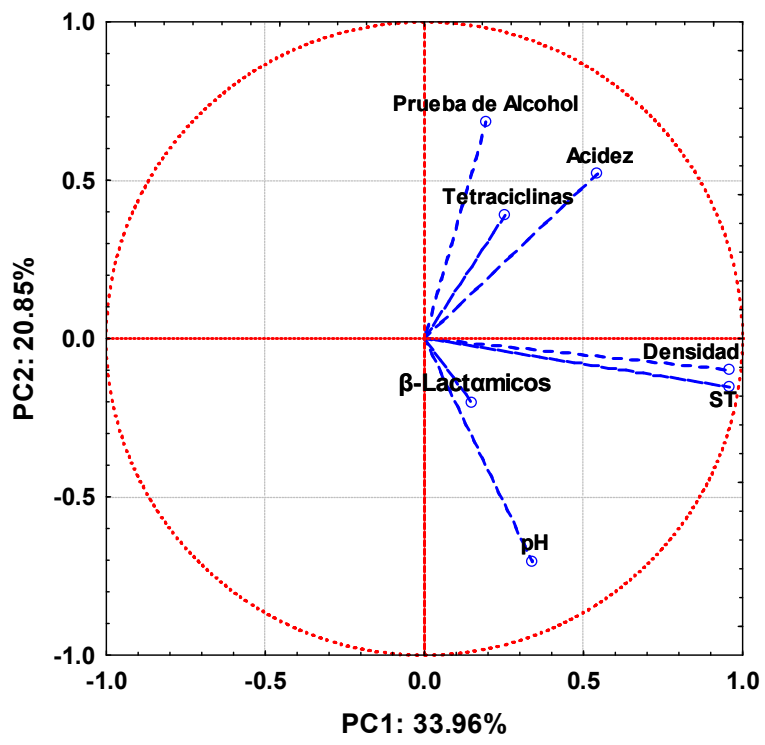

Figura 2. Gráfico de pesos (Loadings) para PC1 y PC2 


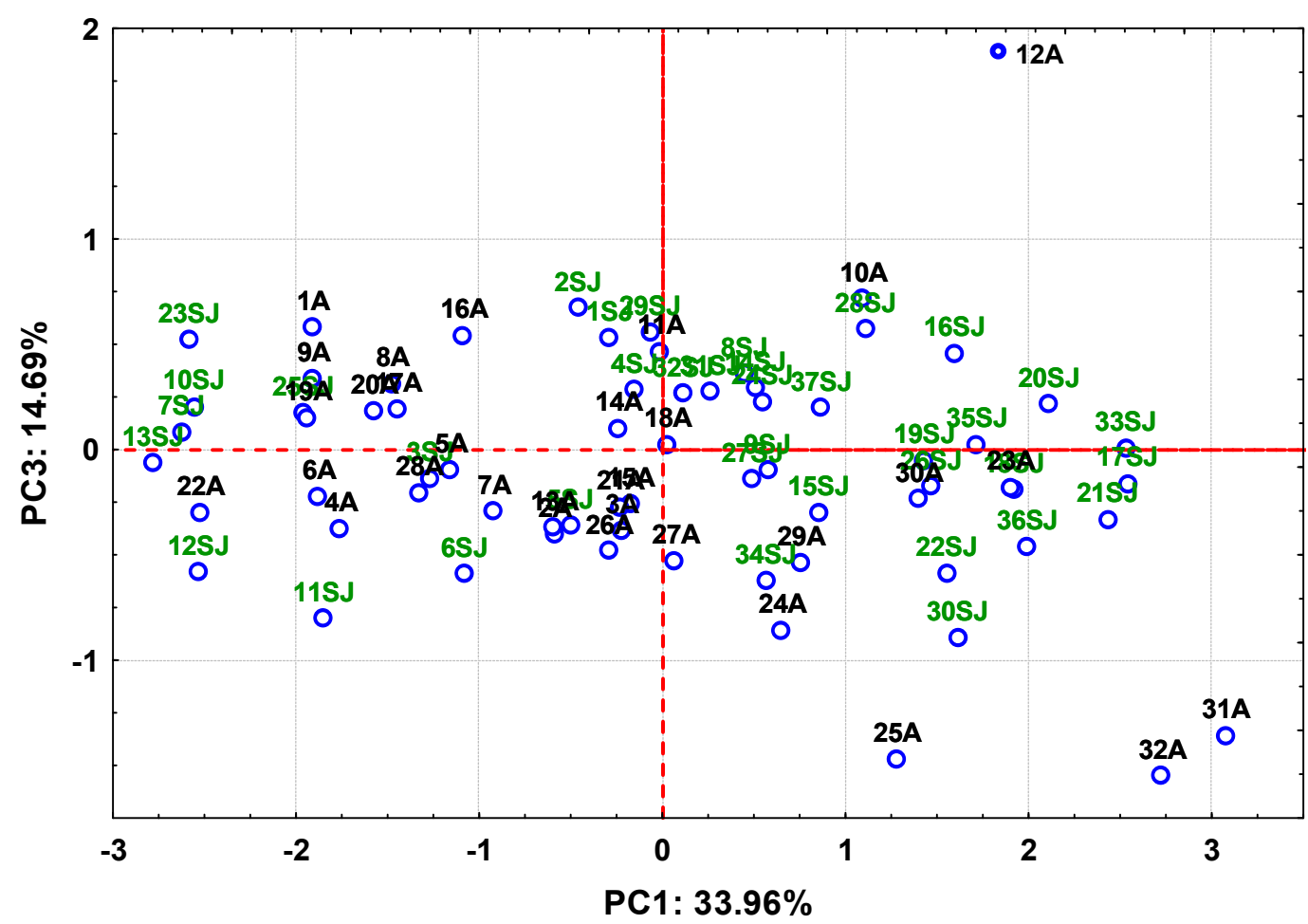

Figura 3. Gráfico de puntuaciones (Scores) para PC1 y PC3

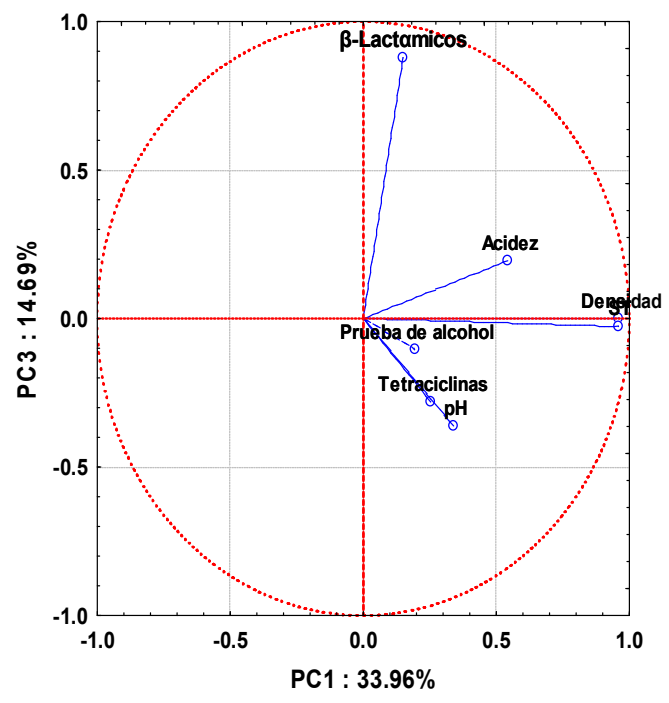

Figura 4. Gráfico de pesos (Loadings) para PC1 y PC3
En el análisis de componentes principales (ACP) se evidencia que el componente principal 1-(PC1) agrupa a las variables densidad, acidez y ST cuyos factores de carga son mayores a 0.4 (Cuadro 5). Para el PC2, las variables Prueba de alcohol y Tetraciclinas son representativas, y para el PC3 es la variable $\beta$-lactámicos. El gráfico de puntuaciones (scores) para PC1 y PC2 (Figura 1) evidenció que la mayoría de muestras de leche provenientes de los comités del distrito de San Jerónimo presentaron altos valores para los parámetros densidad, acidez, pH y ST (valores de $\mathrm{PC} 1$ positivos), lo que se contrasta con la gráfica de pesos (Figura 2), puesto que estos parámetros se encuentran a lado derecho de la gráfica. No obstante, algunos comités de este distrito se encuentran al lado negativo de PC1, posiblemente debido a su 
cercanía territorial a los comités del distrito de Andahuaylas. Por otra parte, en cuanto se refiere a PC2 (Prueba de alcohol y tetraciclinas) se evidencia que los comités que reportaron positivos a tetraciclinas se encuentran en lado superior positivo, tal como se observa en el Cuadro 2.

En la Figura 3 se observa que el Comité 12A (Coñeccpuquio II) reportó positivo a $\beta$ lactámicos, el cual está representado por el PC3 en la Figura 4, aunque este no representa significativamente a los demás parámetros por encontrarse cerca al eje central.

\section{Conclusiones}

- El 10.1 y $1.4 \%$ de los Comités de Vaso de Leche de los distritos de San Jerónimo y Andahuaylas, en Apurímac, Perú, utilizan leche con residuos de antibióticos derivados de tetraciclinas y $\beta$-lactámicos, respectivamente.

- La densidad, acidez, pH y sólidos totales de la leche de la mayoría de los Comités de Vaso de Leche se encuentran fuera los rangos establecidos en el Decreto Supremo $\mathrm{N}^{\circ}$ 007-2017-MINAGRI, Art. 8 y CODEX Alimentarius, sobre todo la leche utilizada por los Comités del distrito de Andahuaylas.

\section{Literatura Citada}

1. Alvarado C, Zerpa G, Meléndez B, Giménez O, Vivas I. 2006. Uso de la prueba del alcohol en la estimación de la estabilidad proteica en leche de un rebaño Holstein de la zona central de Venezuela. En: XIII Congreso Venezolano de Producción e Industria Animal. Venezuela.

2. Aroca N. 2016. Detección cualitativa de residuos de antibióticos en leche cruda comercializada en el Cantón Naranjal provincia del Guayas. Tesis de Médico Veterinario Zootecnista. Machala, Ecuador: Univ. Técnica de Machala. 52 p.
3. Briones O, Peña E. 2018. Programas sociales y su contribución al desarrollo social Perú 2000 - 2015. Tesis de Economista. Lima, Perú: Univ. Inca Garcilaso de la Vega. 142 p.

4. Brunton L, Knollmann BC, Chabner $B A$. 2011. Las bases farmacológicas de la terapéutica. $12^{\circ}$ ed. México DF: McGraw-Hill Interamericana. 2061 p.

5. Camacho LM, Cipriano M, Cruz B, Gutierrez I, Hernández PE, Peñaloza I, Nambo O. 2010. Residuos de antibioticos en leche cruda comercializada en la Región de Tierra Caliente, de Guerrero, México. REDVET 11(2). [Internet]. Disponible en: https:// www.redalyc.org/pdf/636/63613118006.pdf

6. Camos PL. 2018. El efecto de los programas sociales sobre la desnutrición crónica infantil en el Perú. Tesis de Economista. Lima, Perú: Pontificia Universidad Católica del Perú. 101 p.

7. Carrasco JP, Obando AS. 2013. Determinación de residuos de antibióticos y peróxidos de hidrogeno $\left(\mathrm{H}_{2} \mathrm{O}_{2}\right)$ en leche fresca comercializada en el distrito de Chiclayo, mayo-noviembre 2013. Tesis de Biólogo. Chiclayo, Perú: Univ. Nacional Pedro Ruiz Gallo. 51 p.

8. Chávez L. 2013. Determinación de residuos antimicrobianos en leche fresca entera utilizada para la elaboración de productos lácteos mediante el método Delvotest, enero-diciembre del 2012. Tesis de Químico Farmacéutico. Trujillo, Perú: Universidad Nacional de Trujillo. $31 \mathrm{p}$.

9. CODEX Alimentarius. 2011. Leche y productos lacteos. $2^{\circ}$ ed. Roma, Italia; FAO/OMS. 259 p.

10. Diez-Canseco F, Saavedra-Garcia L. 2017. Programas sociales y reducción de la obesidad en el Perú: reflexiones desde la investigación. Rev Per Med Exp Salud Pública 34: 105-112. doi: 10.17843/ rpmesp.2017.341.2772

11. Fernández D. 2012. Evaluación de los métodos de unión a receptores proteicos para la detección de antibióticos en la leche cruda de cabra. Tesis de Maes- 
tría. Valencia, España: Univ. Politécnica de Valencia. $50 \mathrm{p}$.

12. Guerrero DM, Motta R, Gamarra G, Benavides ER, Roque M, Salazar ME. 2009. Detección de residuos de antibióticos $\beta$-lactámicos y tetraciclinas en leche cruda comercializada en el Ca1lao. Cienc Inv 12: 79-82

13. Gutiérrez R, Noa M, Díaz G, Vega S, González M, Prado G. 2005. Determination of the presence of 10 antimicrobial residues in Mexican pasteurized milk. Interciencia 30: 291-294.

14. Llanos GA. 2002. Determinación de residuos de antibióticos en la leche fresca que consume la población de Cajamarca. Rev Amazónica Inv 2: 35-43.

15. Máttar S, Calderón A, Sotelo D, Sierra M, Tordecilla G. 2009. Detección de antibióticos en leches: un problema de salud pública. Rev Salud Pública 11: 579-590. doi: 10.1590/S0124-00642009000400009

16. [MINAGRI] Ministerio de Agricultura y Riego. 2017. Decreto Supremo que aprueba el Reglamento de la Leche y Productos Lácteos. Lima, Perú: MINAGRI. Decreto Supremo No0072017.34 p. [Internet]. Disponible en: http:/ /www.minagri.gob.pe/

17. Molina MP, Berruga I, Molina A. 2010. La presencia de residuos de antibióticos en la leche de oveja: medidas de control y método de elección. Pequeños Rumiantes 11: 13-22.

18. Monge R, Arias L, Ellner R. 1993. Contamination of bovine milk with residues of inhibitory substances in Costa Rica. Rev Biol Trop 41: 855-856.
19. Noa-Lima E, Noa M, González D.G, Landeros P, Reyes W. 2009. Evaluación de la presencia de residuos de antibióticos y quimioterapéuticos en leche en Jalisco, México. Rev Salud Anim 31:29-33.

20. [NTP] Norma Técnica Peruana. 2003. Leche y productos lácteos. Leche cruda: Lima Perú: NTP 202.001. 13 p.

21. Ortiz C, Vera R, Cayro J. 2008. Frecuencia de $\beta$-lactámicos y tetraciclinas en leche fresca en la Cuenca de Arequipa. Rev Inv Vet Perú 19: 140-143.

22. Prado G, Carabias R, Rodríguez E, Herrero E. 2002. Presencia de residuos y contaminantes en leche humana. Rev Española Salud Pública 76: 121-132.

23. Ramírez A, Gutiérrez R, Gonzales $C$, Escobar I, Castro G, Diaz G, Noa M. 2001. Detección de antibióticos en leche comercializada en la ciudad de México. Rev Salud Anim 23: 37-41.

24. Rodríguez L, Mendoza G 2011. Residuos de antibióticos (tetraciclinas y betalactámicos) en leche entera de acopios de Matiguas - Matagalpa, mediante la prueba de Beta Star Combo en el periodo de noviembre del 2010 a abril 2011. Tesis de Médico Veterinario. Managua, Nicaragua: Univ. Nacional Agraria. 31 p.

25. Salas P, Calle S, Falcon N, Pinto C, Espinoza J. 2013. Determinación de residuos de antibióticos betalactámicos mediante un ensayo inmunoenzimático en leche de vacas tratadas contra mastitis. Rev Inv Vet Perú 24: 252-255. doi: 10.15381/rivep.v24i2.2519 